\title{
Cationic Ring-Opening Oligomerization of the Two Stereoisomers of 4-Bromo-6,8-dioxabicyclo[3.2.1]
}

\author{
Masahiko OKADA, Hiroshi Sumitomo, Kanako ITO, \\ Setsuko GoTo, and Miharu ATSUMI \\ Faculty of Agriculture, Nagoya University, \\ Chikusa, Nagoya 464, Japan
}

(Received June 27, 1987)

\begin{abstract}
Ring-opening oligomerization of the two stereoisomers of a bicyclic lactone 4bromo-6,8-dioxabicyclo[3.2.1] octan-7-one was investigated in dichloromethane and chloroform at -40 and $0^{\circ} \mathrm{C}$ using antimony pentachloride, phosphorus pentafluoride, and trifluoromethanesulfonic acid as initiators. The axial isomer (5a) showed a tendency to cyclodimerize, particularly at higher temperature, whereas the equatorial isomer (5e) was much less reactive and it afforded only a small amount of cyclic oligomers. Reductive debromination using tri- $\boldsymbol{n}$-butylstannane converted the cyclic dimer (6) of $5 \mathbf{a}$ to the cyclic dimer (2) of 6,8-dioxabicyclo[3.2.1] octan-7-one (1), indicating that 6 was composed of a pair of the enantiomeric monomeric units. The specific formation of the cyclic dimer $\mathbf{6}$ from the axial isomer 5a was interpreted in terms of the participation of bromonium ions in the propagation.
\end{abstract}

KEY WORDS Ring-Opening Oligomerization / Bicyclic Lactone / Stereoisomer / 4-Bromo-6,8-dioxabicyclo[3.2.1]octan-7-one / Cyclic Dimer / Bromonium Ion / Neighboring Group Participation /

Ring-opening polymerization of cyclic compounds is often accompanied by the formation of cyclic oligomers of different ring sizes, and in some cases, cyclic oligomers are produced as major products. ${ }^{1-3}$ Previously, we found specific formation of 10-, 20-, and 25-membered macrocyclic oligoesters (cyclic dimer (2), tetramer (3), and pentamer (4)) in the cationic ring-opening polymerization of a bicyclic lactone, 6,8-dioxabicyclo[3.2.1]octan-7-one (1) ${ }^{4-7}$ From one of the antipodes of 1 , only the cyclic tetramer and pentamer were produced. These oligomers were exclusively or highly selectively produced under proper selection of reaction conditions, particularly solvent and temperature. Interestingly, cyclic dimer $\mathbf{2}$ is a meso compound consisting of a pair of the enantiomeric monomeric units, while cyclic tetramer 3 and cyclic pentamer $\mathbf{4}$ derived from the racemic monomer 1 are racemic mixtures, that is, they are equimolar mixtures of the corresponding cyclic oligomers of the optically active monomers, $(\mathbf{1 R})_{n}$ or $(\mathbf{1 S})_{n}(n=$ $4,5)$.

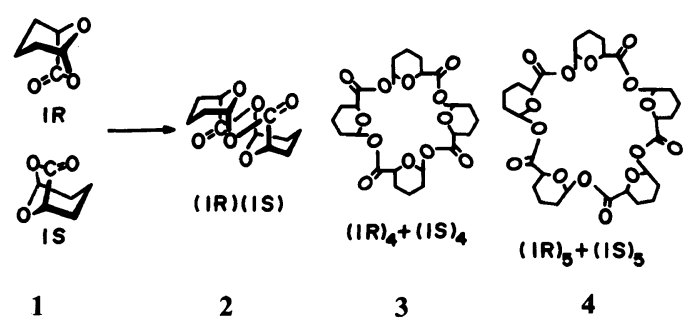

These oligoesters are composed of alternating tetrahydopyran ring and ester moieties, and therefore bear structural resemblance to a naturally occurring antibiotic nonactin. Nonactin is a 32-membered tetrolide containing tetrahydropyran rings, and selectively enhances the transport of potassium ion through cell membranes. ${ }^{8}$ The 20- and 25-membered oligoesters actually act as ion carriers for the 
transport of metal ions through organic liquid membranes as well as through acetyl cellulose membranes. ${ }^{9,10}$ These oligoesters are also capable of forming stable complexes with neutral organic molecules such as acetonitrile and acetone. ${ }^{11-13}$

The formation of these cyclic oligoesters is specific to the bicyclic oxalactone 1 having a bicyclo[3.2.1]octane skeleton, because its structural isomers having a bicyclo[2.2.2]octane such as 2,5-dioxabicyclo[2.2.2]octan-3one and 2,6-dioxabicyclo[2.2.2]octan-3-one did not produce cyclic oligomers but linear polyesters under similar reaction conditions. ${ }^{14-16}$

During the course of a series of studies on the ring-opening polymerization of bicyclic lactones, we investigated the cationic oligomerization of the axial and equatorial isomers (5a and 5e) of 4-bromo-6,8-dioxabicyclo[3:2.1] octan-7-one. These stereoisomers were chosen in order to clarify reaction control by neighboring group participation of a bromine substituent in the cyclooligomerization on the one hand, and to design functional cyclic oligomers through appropriate chemical modifications on the other hand. The present paper deals with significantly different behavior between these two stereoisomers in their cationic ring-opening oligomerization.

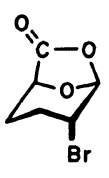

$5 a$

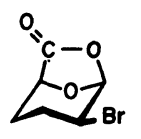

$5 \mathbf{e}$

\section{EXPERIMENTAL}

Preparation of Stereoisomers of 4-Bromo-6,8dioxabicyclo[3.2.1]octan-7-one (5a and 5e)

Sodium 3,4-dihydro-2 $H$-pyran-2-carboxylate $(31.5 \mathrm{~g}, 0.21 \mathrm{~mol})$, anhydrous sodium carbonate $(12.0 \mathrm{~g}, 0.11 \mathrm{~mol})$, and dimethylformamide $(300 \mathrm{ml})$ were placed in a $500 \mathrm{ml}$ three-necked flask. Bromine $(36.0 \mathrm{~g}, 0.23 \mathrm{~mol})$ was added dropwise to the mixture at $30^{\circ} \mathrm{C}$ with vigorous stirring. After having been stirred at $30^{\circ} \mathrm{C}$ for $3 \mathrm{~h}$, the reaction mixture was poured into water $(1000 \mathrm{ml})$. The mixture was extracted with four $150 \mathrm{ml}$ portions of benzene. The combined benzene extracts were washed with two $150 \mathrm{ml}$ portions of water and dried over anhydrous magnesium sulfate. The mixture was filtered and the filtrate was subjected to rotary evaporation to yield a mixture of $5 \mathbf{a}$ and $5 \mathbf{e}\left(4: 3\right.$, by ${ }^{13} \mathrm{C}$ NMR) as a colorless solid. The mixture was dissolved in diethyl ether, and the solution was allowed to stand in a refrigerator to afford colorless crystals. They were separated by filtration, and recrystallized twice from diethyl ether to yield the axial isomer 5a. From the filtrate, a colorless solid was obtained by rotary evaporation. It was chromatographed on a silica gel column with a mixture of ethyl acetate and $n$-hexane $(1: 3, \mathrm{v} / \mathrm{v})$ as eluent $\left(R_{f}=\right.$ 0.66 for $5 \mathrm{a}$ and $R_{f}=0.51$ for $5 \mathrm{e}$ ). The fractions containing 5e were combined and evaporated to dryness. The residue was repeatedly recrystallized from $n$-hexane.

5a: $\mathrm{mp}, 102.5-103^{\circ} \mathrm{C}$; IR $(\mathrm{KBr}) \mathrm{cm}^{-1}, 1790$ $v_{\mathrm{C}=\mathrm{o}}, 1200 v_{\mathrm{C}-\mathrm{o}-\mathrm{C}}(\gamma$-lactone $), 1150,1095$ $v_{\mathrm{C}-\mathrm{O}-\mathrm{C}}($ acetal $), 700 v_{\mathrm{C}-\mathrm{Br}} ;{ }^{1} \mathrm{H} \mathrm{NMR}\left(\mathrm{CDCl}_{3}\right)$, $\delta 5.89$ (br s, $1 \mathrm{H}, \mathrm{H}-5), 4.47$ (m, 1H, H-1), 4.15 $\left(\mathrm{dd}, J_{4,5}=1.8 \mathrm{~Hz}, J_{3 \mathrm{a}, 4}=4.6 \mathrm{~Hz}, 1 \mathrm{H}, \mathrm{H}-4\right)$, $2.46-2.68\left(\mathrm{~m}, 1 \mathrm{H}, \mathrm{H}-3_{\mathrm{e}}\right), 2.02-2.46(\mathrm{~m}, 2 \mathrm{H}$, $\left.\mathrm{H}-2_{\mathrm{a}}, \mathrm{H}-3_{\mathrm{a}}\right), 1.60-1.84 \mathrm{ppm}\left(\mathrm{m}, 1 \mathrm{H}, \mathrm{H}-2_{\mathrm{e}}\right)$; ${ }^{13} \mathrm{C}$ NMR data are given in Table III.

\section{Reductive Debromination}

A sample of oligomer $(0.15 \mathrm{~g}, 0.72$ base$\mathrm{mmol})$, azobis(isobutyronitrile) $(0.02 \mathrm{~g}, 0.12$ mmol), tetrahydrofuran $(20 \mathrm{ml})$ and tri- $n$ butylstannane $(0.50 \mathrm{~g}, 1.7 \mathrm{mmol})$ were charged in this order in a three-necked flask equipped with a condenser and a nitrogen inlet. The mixture was refluxed with magnetic strirring for $52 \mathrm{~h}$ under a stream of nitrogen. The reaction mixture was concentrated by removing tetrahydrofuran under reduced pressure. The subsequent addition of $n$-hexane to the mixture afforded a pale yellow precipitate. The 
precipitate was washed with $n$-hexane repeatedly to remove tri- $n$-butylbromostannane formed in the reaction and also unreacted tri$n$-butylstannane. The product thus obtained was dried under reduced pressure.

When a sample of 5a having a high IR absorbance ratio $A^{1740} / A^{1770}$ was reductively debrominated, colorless crystals deposited on the wall of the flask. They were identified as the cyclic dimer 2 by IR, ${ }^{1} \mathrm{H}$ and ${ }^{13} \mathrm{C}$ NMR spectroscopy: IR $(\mathrm{KBr}) \mathrm{cm}^{-1}, 1735 v_{\mathrm{C}=\mathrm{O}}, 1232$ $v_{\mathrm{C}-\mathrm{o}-\mathrm{C}}\left(\right.$ ester), $1120,1040 v_{\mathrm{C}-\mathrm{o}-\mathrm{C}}\left(\right.$ acetal); ${ }^{1} \mathrm{H}$ NMR $\left(\mathrm{CDCl}_{3}\right), \delta 6.36$ (s, 1H, OCHO), 4.39 (d, $1 \mathrm{H}, \quad \mathrm{OCHC}=\mathrm{O}), \quad 1.6-2.4 \mathrm{ppm} \quad(\mathrm{m}, \quad 6 \mathrm{H}$, $\left.\mathrm{CH}_{2} \mathrm{CH}_{2} \mathrm{CH}_{2}\right) ;{ }^{13} \mathrm{C}$ NMR $\left(\mathrm{CDCl}_{3}\right), \delta 171.09$ $(\mathrm{C}=\mathrm{O}), 91.74$ (OCHO), $70.26(\mathrm{O} \underline{\mathrm{C}} \mathrm{HC}=\mathrm{O})$, $27.34\left(\mathrm{OCHC}_{2}\right), \quad 24.71 \quad\left(\underline{\mathrm{CH}}_{2} \mathrm{CHC}=\mathrm{O}\right)$, $13.82 \mathrm{ppm}\left(\mathrm{CH}_{2} \mathrm{CH}_{2} \mathrm{CH}_{2}\right)$.

\section{Characterization}

${ }^{1} \mathrm{H}$ and ${ }^{13} \mathrm{C}$ NMR spectra were recorded on JEOL FX-200 and FX-100 instruments operating at 200 and $100 \mathrm{MHz}\left({ }^{1} \mathrm{H}\right)$, and 50 and $25 \mathrm{MHz}\left({ }^{13} \mathrm{C}\right)$, respectively, on solutions in deuteriochloroform and perdeuteriodimethyl sulfoxide. Tetramethylsilane was used as internal reference. IR spectra were measured by a JASCO A-3 spectrometer on KBr disks. Gel permeation chromatograms were taken by a JEOL Trirotor gel permeation chromatograph (column, JASCO JSP 101, $50 \mathrm{~cm}$; eluent, chloroform; polystyrene standard).

\section{RESULTS AND DISCUSSION}

\section{Synthesis of Monomers}

The stereoisomers $\mathbf{5 a}$ and $\mathbf{5 e}$ were readily prepared in one step by bromination of sodium 3,4-dihydro- $2 H$-pyran-2-carboxylate in dimethylformamide. The reaction product was obtained as a mixture of $\mathbf{5 a}$ and $\mathbf{5 e}$. The ratio of $5 \mathbf{a} / \mathbf{5} \mathbf{e}$ changed significantly, depending on the reaction temperature; $\mathbf{5 a} / \mathbf{5 e}=4 / 1$ at $-20^{\circ} \mathrm{C}$ and $5 \mathrm{a} / \mathbf{5 e}=4 / 3$ at $30^{\circ} \mathrm{C}$. The separation of these stereoisomers was successfully achieved by a combination of recrystallization and column chromatography, as described in the Experimental Part. The stereoisomers thus obtained were characterized by IR, ${ }^{1} \mathrm{H}$ and ${ }^{13} \mathrm{C}$ NMR spectroscopy, and elemental analysis.

\section{Polymerization of the Axial Isomer 5a}

Cationic polymerization of $\mathbf{5 a}$ was carried out in dichloromethane and chloroform at 0 and $-40^{\circ} \mathrm{C}$, chiefly with antimony pentachloride as the initiator. The polymerization system became heterogeneous as the polymerization proceeded. Some of the results of the polymerization are summarized in Table I.

Antimony pentachloride induced the po-

Table I. Polymerization of 4(a)-bromo-6,8-dioxabicyclo[3.2.1]octan-7-one (5a)

\begin{tabular}{|c|c|c|c|c|c|c|}
\hline Monomer & & Solvent ${ }^{b}$ & Temp & Time & Yield & $A^{1740 c}$ \\
\hline g & & $\mathrm{ml}$ & ${ }^{\circ} \mathrm{C}$ & $\mathrm{h}$ & $\%$ & $A^{1770}$ \\
\hline 0.50 & $\mathrm{SbCl}_{5}$ & DM 2.5 & 0 & 3 & 40 & 5.5 \\
\hline 0.50 & $\mathrm{SbCl}_{5}$ & DM 2.5 & 0 & 24 & 52 & 6.8 \\
\hline 0.50 & $\mathrm{SbCl}_{5}$ & DM 2.5 & -40 & 3 & 4 & 0.20 \\
\hline 0.50 & $\mathrm{SbCl}_{5}$ & DM 2.5 & -40 & 24 & 37 & 2.6 \\
\hline 0.50 & $\mathrm{SbCl}_{5}$ & CF 3.0 & 0 & 48 & 50 & 4.7 \\
\hline 0.50 & $\mathrm{SbCl}_{5}$ & CF 3.0 & -40 & 24 & 20 & 0.83 \\
\hline 1.50 & $\mathrm{SbCl}_{5}$ & CF $\quad 9.0$ & -40 & 48 & 37 & 1.2 \\
\hline 1.50 & $\mathrm{CF}_{3} \mathrm{SO}_{3} \mathrm{H}$ & CF $\quad 9.0$ & -40 & 48 & 1.5 & 1.1 \\
\hline
\end{tabular}

a $5 \mathrm{~mol} \%$ to monomer.

b DM, dichloromethane; CF, chloroform.

c Absorbance ratio of the carbonyl stretching bands in IR spectrum. 
lymerization of $5 \mathbf{a}$ under the reaction conditions examined to afford colorless powdery products in yields up to $52 \%$. Trifluoromethanesulfonic acid was much less effective. The products showed only limited solubilities in common organic solvents. They dissolved

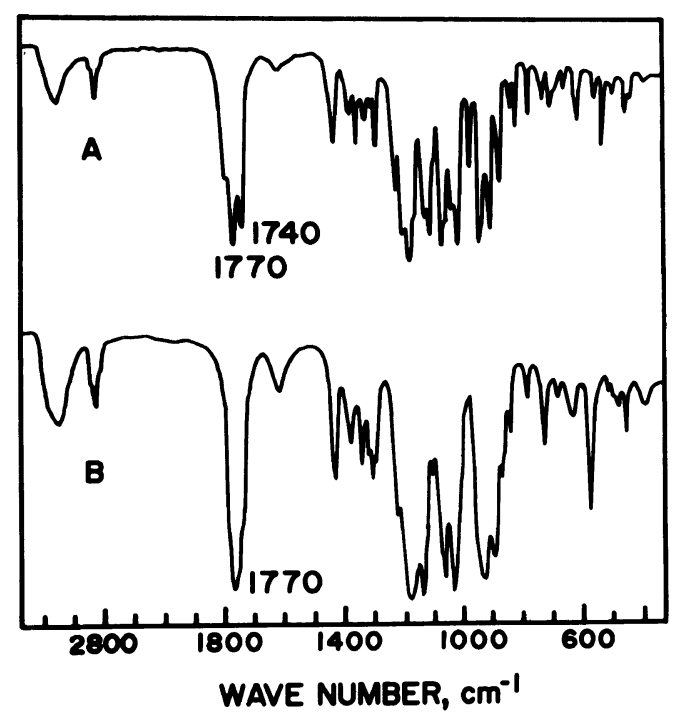

Figure 1. IR spectra of the oligomers of 4(a)- and 4(e)bromo-6,8-dioxábicyclo[3.2.1]octan-7-one ( $\mathrm{KBr})$. A, the axial isomer (5a); $\mathbf{B}$, the equatorial isomer (5e). Reaction conditions: initiator, antimony pentachloride; solvent, chloroform (5a) or dichloromethane (5e); temperature, $-40^{\circ} \mathrm{C}$; time, $24 \mathrm{~h}$. slightly in dimethyl sulfoxide and much less in chloroform $(<0.1 \%)$. The products did not melt below $300^{\circ} \mathrm{C}$.

The polymerization products of $5 \mathbf{a}$ exhibited two infrared carbonyl stretching absorptions at 1740 and $1770 \mathrm{~cm}^{-1}$ (Figure 1, A). This is in sharp contrast to the polymerization products of 5e showing a single carbonyl absorption at $1770 \mathrm{~cm}^{-1}$ (Figure 1, B). The relative intensities of these bands varied, depending on the reaction conditions, as clearly demonstrated by the absorbance ratio $A^{1740} / A^{1770}$ in the last column of Table I. The ratio increased with conversion at a given temperature and also with rise in polymerization temperature. On the basis of the spectral data of the cyclic oligomers of $1\left(v_{\mathrm{C}=\mathrm{o}} \mathrm{cm}^{-1} ; 2,1735 ; 3,1760 ; \mathbf{4}\right.$, $1761)$, it was inferred that the polymerization product of $5 \mathbf{a}$ contains a cyclic dimer having a skeleton similar to that of 2 .

Figure 2 shows the ${ }^{13} \mathrm{C}$ NMR spectrum of the product $\left(A^{1740} / A^{1770}=1.2\right)$ obtained by the polymerization of $5 \mathbf{a}$ in chloroform at $-40^{\circ} \mathrm{C}$. The spectrum is not clear due to the poor solubility of the sample in dimethyl sulfoxide$d_{6}$, but there appear two sets of signals, which indicate the coexistence of two components in comparable amounts. From a comparison of the chemical shifts with those for the cyclic

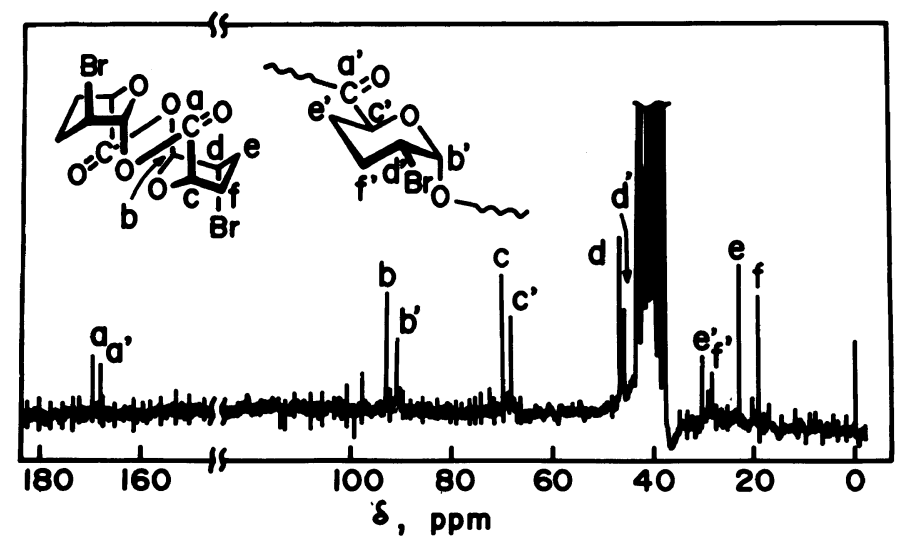

Figure 2. ${ }^{13} \mathrm{C}$ NMR spectrum of the product obtained by the polymerization of $4(\mathrm{a})$-bromo-6,8dioxabicyclo[3.2.1] octan-7-one (5a) in chloroform at $-40^{\circ} \mathrm{C}$ with antimony pentachloride as the initiator. Solvent, dimethyl sulfoxide- $d_{6}$; temperature, $70^{\circ} \mathrm{C}$; internal. reference, tetramethylsilane; $25 \mathrm{MHz}$. 
oligomers of 1 , the set of the signals with higher intensities $(\mathrm{a}-\mathrm{f})$ was reasonably assigned to the cyclic dimer, and the other set of the signals with lower intensities $\left(a^{\prime}-f^{\prime}\right)$ to other higher oligomers consisting of the regular structural unit as illustrated in Figure 2.

Quantitative analysis by gel permeation chromatography was not feasible because the polymerization products did not completely dissolve in chloroform. Qualitatively, however, there were three components corresponding to dimer, tetramer, and polymer in the products obtained in dichloromethane at $0^{\circ} \mathrm{C}$, whereas there were three components corresponding to dimer, tetramer, and pentamer in the products prepared in chloroform at $-40^{\circ} \mathrm{C}$. Therefore, the carbonyl absorption at $1770 \mathrm{~cm}^{-1}$ is assignable to the tetramer and pentamer (probably cyclic) and/or polymer possessing the identical structural unit given in Figure 2. Among these oligomers, only the cyclic dimer could be isolated by gel permeation chromatography and characterized. The spectroscopical as well as analytical data are given in EXPERIMENTAL.

In order to confirm the structures of the oligomers, the reductive debromination of the products using tri- $n$-butylstannane was undertaken in tetrahydrofuran in the presence of a small amount of azobis(isobutyronitrile). Tri$n$-butylstannane has been successfully employed for the reductive dechlorination of poly(vinyl chloride) ${ }^{17,18}$ as well as for the reductive debromination of polymers derived from 4-bromo-6,8-dioxabicyclo[3.2.1]octane. ${ }^{19}$ Dehalogenation by this reagent takes place via a radical mechanism, and hence it does not require the energetically unfavorable flipping of a tetrahydropyran ring, unlike $S_{N} 2$ reactions.

A sample having an $A^{1740} / A^{1770}$ ratio of 6.8 was reductively debrominated to yield colorless crystals. The IR, ${ }^{1} \mathrm{H}$, and ${ }^{13} \mathrm{C}$ NMR spectral data were in perfect agreement with those for cyclic dimer $\mathbf{2}$. The structure of $\mathbf{2}$ has been established by X-ray analysis. ${ }^{11}$ It is composed of a pair of the enantiomeric monomeric units and all four bonds attached to the two tetrahydropyran rings are axially oriented. Therefore, the major oligomerization product of 5a showing a carbonyl stretching absorption at $1740 \mathrm{~cm}^{-1}$ is proved to be the cyclic dimer (6) possessing the skeleton identical to that of 2, that is, consisting of a pair of the enantiomeric monomeric units.

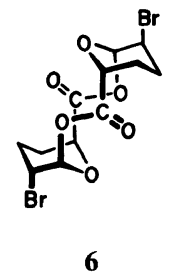

A sample with an $A^{1740} / A^{1770}$ ratio of 1.0 , however, gave on reductive debromination an unidentified solid which was hardly soluble in organic solvents, along with cyclic dimer 2. The IR spectrum of the solid did not show any carbonyl absorption in the region of 1700 $1800 \mathrm{~cm}^{-1}$ but strong carbonyl absorption at $1590 \mathrm{~cm}^{-1}$. This suggests occurrence of some cleavage reactions. Accordingly, confirmative evidence for the structures of the other components exhibiting a carbonyl stretching band at $1770 \mathrm{~cm}^{-1}$ could not be obtained.

\section{Polymerization of the Equatorial Isomer 5e}

Polymerization of $5 \mathbf{e}$ was undertaken in dichloromethane at -40 and $0^{\circ} \mathrm{C}$ with three different initiators. Some of the results are listed in Table II. In comparison with $\mathbf{5 a}, \mathbf{5 e}$ displayed much less polymerizability. Moreover, prolonged polymerization of $5 \mathbf{e}$ caused side reactions to yield products of complicated structures.

The difference in the reactivity between $5 \mathbf{a}$ and $\mathbf{5} \mathbf{e}$ is in qualitative agreement with the previous observation that the axial isomer of 4-bromo-6,8-dioxabicyclo[3.2.1]octane was more reactive than the equatorial isomer in their cationic copolymerization. ${ }^{20}$ The lower polymerizability of $5 \mathbf{e}$ seems to arise from at 
Table II. Polymerization of 4(e)-bromo-6,8-dioxabicyclo[3.2.1]octan-7-one (5e)

\begin{tabular}{|c|c|c|c|c|c|c|}
\hline Monomer & \multirow{2}{*}{ Initiator $^{a}$} & \multirow{2}{*}{$\frac{\text { Solvent }^{\mathrm{b}}}{\mathrm{ml}}$} & \multirow{2}{*}{$\frac{\text { Temp }}{{ }^{\circ} \mathrm{C}}$} & \multirow{2}{*}{$\frac{\text { Time }}{\mathrm{h}}$} & \multirow{2}{*}{$\begin{array}{c}\text { Yield } \\
\%\end{array}$} & \multirow{2}{*}{$\begin{array}{l}\text { Major } \\
\text { product }\end{array}$} \\
\hline g & & & & & & \\
\hline 0.50 & $\mathrm{SbCl}_{5}$ & 0.50 & 0 & 3 & 5 & Cyclic \\
\hline 0.50 & $\mathrm{SbCl}_{5}$ & 0.50 & 0 & 24 & 27 & Linear \\
\hline 0.50 & $\mathrm{SbCl}_{5}$ & 0.50 & 0 & 120 & 18 & Linear \\
\hline 0.50 & $\mathrm{SbCl}_{5}$ & 2.5 & -40 & 24 & 6 & Cyclic \\
\hline 2.00 & $\mathrm{PF}_{5}$ & 10.0 & -40 & 24 & 5 & Cyclic \\
\hline 2.00 & $\mathrm{CF}_{3} \mathrm{SO}_{3} \mathrm{H}$ & 10.0 & -40 & 24 & 0.3 & Cyclic \\
\hline
\end{tabular}

a $5 \mathrm{~mol} \%$. to monomer.

b Dichloromethane.

c Cyclic, cyclic oligomers; linear, linear oligomers. See the text.

Table III. ${ }^{13} \mathrm{C}$ NMR chemical shifts of the stereoisomers (5a and 5e) of 4-bromo-6,8-dioxabicyclo[3.2.1] octan-7-one and their oligomers $^{\mathrm{a}}$

\begin{tabular}{|c|c|c|c|c|c|}
\hline \multirow{2}{*}{ Assignment } & \multicolumn{3}{|c|}{$5 a$} & \multicolumn{2}{|c|}{$5 e$} \\
\hline & Monomer $^{\mathbf{b}}$ & Dimer $^{\mathbf{c}}$ & Oligomer $^{d}$ & Monomer $^{b}$ & Oligomer $^{d}$ \\
\hline $\mathrm{C}=\mathrm{O}$ & 171.63 & 169.02 & 167.47 & 171.50 & 166.75 \\
\hline ОСНO & 102.77 & 92.35 & 90.24 & 103.69 & 91.32 \\
\hline $\mathrm{O} \underline{\mathrm{CHC}}=\mathrm{O}$ & 72.41 & 69.28 & 67.75 & 71.40 & 68.46 \\
\hline $\mathrm{CHBr}$ & 42.30 & 46.38 & 46.15 & 42.97 & 46.20 \\
\hline $\mathrm{CH}_{2} \mathrm{CH}_{2} \mathrm{CHBr}$ & 25.63 & 22.90 & 29.88 & 27.77 & 26.31 \\
\hline $\mathrm{CH}_{2} \mathrm{C} H \mathrm{C}=\mathrm{O}$ & 20.61 & 19.26 & 28.18 & 26.46 & 21.83 \\
\hline
\end{tabular}

a $\delta$, ppm; solvent, dimethyl sulfoxide- $d_{6}$; temp, $70^{\circ} \mathrm{C}$; internal reference, tetramethylsilane; $25 \mathrm{MHz}$.

b Solvent, deuteriochloroform; room temperature; $50 \mathrm{MHz}$.

c Cyclic dimer.

d Cyclic tetramer and/or cyclic pentamer.

least two factors, the absence of the participation of bromonium ions in the polymerization of $5 \mathbf{e}$ as will be discussed in the following section, and the thermodynamically higher stability of the monomer $\mathbf{5 e}$ and the lower stability of its ring-opened structure than those for $5 \mathbf{a}$ and its ring-opened structure. ${ }^{20}$

The polymerization products of $5 \mathbf{e}$ were colorless powders slightly soluble in warm dimethyl sulfoxide. The products obtained at $-40^{\circ} \mathrm{C}$ showed only one strong carbonyl absorption at $1770 \mathrm{~cm}^{-1}$ without any discernible absorption at $1740 \mathrm{~cm}^{-1}$ (Figure 1, B). This is an indication that there was little, if any, cyclic dimer in the products.

The ${ }^{13} \mathrm{C}$ NMR spectrum of the polymer- ization product obtained at $-40^{\circ} \mathrm{C}$ with antimony pentachloride as the initiator showed essentially a set of six signals, although the signal to the noise ratio of the spectrum was considerably low due to the extremely poor solubility of the sample. From a comparison of the chemical shifts with those for $\mathbf{5 e}$ as well as those for the oligomers of $\mathbf{5 a}$ (Table III), it was postulated that the major oligomeric products of $5 \mathbf{e}$ consist of the regular structural unit as depicted in Figure 3.

Figure 3 presents the ${ }^{1} \mathrm{H}$ NMR spectrum of the polymerization product prepared with phosphorus pentafluoride at $-40^{\circ} \mathrm{C}$. The products obtained with other initiators also gave essentially similar ${ }^{1} \mathrm{H}$ NMR spectra. The 


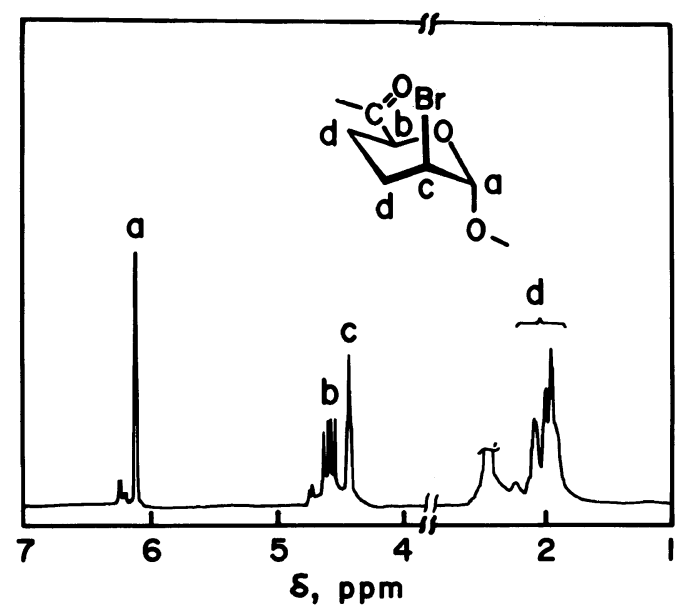

Figure 3. ${ }^{1} \mathrm{H}$ NMR spectrum of the oligomers obtained by the polymerization of 4(e)-bromo-6,8-dioxabicyclo[3.2.1]octan-7-one (5e) in dichloromethane at $-40^{\circ} \mathrm{C}$ with phosphorus pentafluoride as the initiator. Solvent, dimethyl sulfoxide- $d_{6}$; temperature, $70^{\circ} \mathrm{C}$; internal reference, tetramethylsilane; $200 \mathrm{MHz}$.

sharpness of the signals, particularly clear doublet-doublet splittings of the signal $b(\delta$ $4.59 \mathrm{ppm}, J=6.3$ and $8.5 \mathrm{~Hz}$ ) assignable to the methine proton on the carbonyl-bearing carbon, along with the lack of the signals ascribable to end groups, are strongly indicative of the presence of cyclic oligomers composed of the regular structural units drawn in Figure 3. Unfortunately, the major products were hardly soluble in chloroform and therefore, information regarding their ring size could not be obtained by gel permeation chromatography. However, by analogy of the cationic oligomerization behavior of the parent monomer $\mathbf{1}$, it seems likely that cyclic tetramer and/or pentamer were formed in the cationic polymerization of $5 \mathbf{e}$ as well. An attempt to debrominate the oligomers of $\mathbf{5 e}$ with tri- $n$-butylstannane for structural analysis was unsuccessful.

The appearance of a weak doublet at $\delta$ $6.20 \mathrm{ppm}(J=3.4 \mathrm{~Hz})$ and a weak doubletdoublet at $\delta 4.75 \mathrm{ppm}(J=1.4$ and $5.4 \mathrm{~Hz})$ demonstrates the presence of a minor amount of cyclic dimer having the same skeleton as that of 6. In fact, GPC analysis of the chloroform soluble part of the product revealed the presence of components corresponding to cyclic dimer and tetramer.

The oligomerization products obtained at $0^{\circ} \mathrm{C}$ after prolonged reaction showed different and complicated ${ }^{1} \mathrm{H}$ and ${ }^{13} \mathrm{C}$ NMR spectra. In view of the fact that they were readily soluble in dimethyl sulfoxide, these products must be composed of irregular structures resulting from some secondary reactions. In this connection, a linear polymer containing fivemembered oxalactone (1,3-dioxolan-4-one) rings (7) in the backbone chain was obtained in the cationic polymerization of the parent monomer 1 at $0^{\circ} \mathrm{C}$. $^{21}$ This polymer is not formed directly by the ring-opening polymerization of 1 but by the isomerization of the once-formed polyester or oligoester chains.

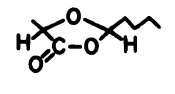

cis<smiles>CC1OC2OC(=O)OC1O2</smiles>

trans 7

Therefore, it appears that also in the present oligomerization, a similar side reaction takes place, although not so rapidly as in the polymerization of 1 , to yield linear oligomeric products. In fact, the IR spectrum of the products showed a strong absorption at $1800 \mathrm{~cm}^{-1}$ characteristic of a 1,3-dioxolan-4one ring, in addition to a weaker absorption at $1770 \mathrm{~cm}^{-1}$. Furthermore, the ${ }^{1} \mathrm{H}$ and ${ }^{13} \mathrm{C}$ NMR spectra gave additional signals assignable to the acetal protons and carbons of cisand trans-2,5-disubstituted-1,3-dioxolan-4-one rings at $\delta 5.88$ and 5.69, and 102.6 and 101.6 ppm, respectively. All these spectroscopical data support that five-membered oxalactone ring structures similar to 7 exist as the major constituent in the reaction products obtained at $0^{\circ} \mathrm{C}$ after prolonged reaction.

\section{Mechanism of Cyclodimerization of $\mathbf{5 a}$}

As described in the foregoing section, cyclic 
dimer 6 of a specific configuration was predominantly formed in the oligomerization of 5a, particularly at higher temperature. The formation of 6 from 5 a differs from the formation of $\mathbf{2}$ from $\mathbf{1}$ in that $\mathbf{6}$ is produced even at very low conversion, whereas $\mathbf{2}$ is formed after the middle stage of the oligomerization. ${ }^{5}$ This difference implies that different mechanisms are operative in the cyclodimerization of $5 \mathbf{a}$ and $\mathbf{1 .}$

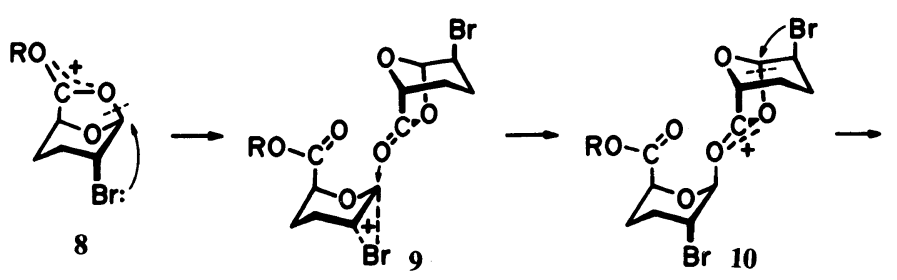

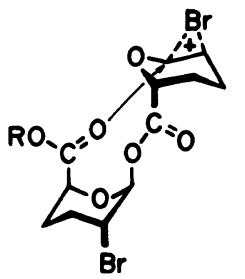

11

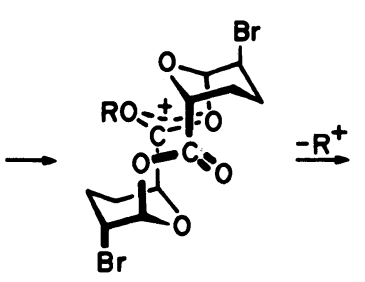

12

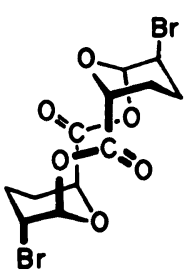

6

Scheme I. Participation of bromonium ions in the formation of cyclic dimer (6) from 4(a)-bromo-6,8dioxabicyclo[3.2.1] octan-7-one (5a).

Scheme I illustrates a possible mechanism of the cyclodimerization of $5 \mathbf{a}$. The growing chain end can be reasonably visualized as an oxonium ion (8), which is converted to the bromonium ion (9) by the neighboring group participation of the axially oriented bromine atom. The formation of the bromonium ion of course competes with the nucleophilic addition of monomer on the acetal carbon. The bromonium ion 9 is then ring-opened by the attack of a monomer having the chirality opposite to that of the terminal unit to generate the oxonium ion (10). The oxonium ion 10 thus formed is again transformed to the bromonium ion (11) to which the nucleophillic addition of the carbonyl oxygen of the penultimate units takes place to yield the 10-membered cyclic oxonium ion (12). Therefrom cyclic dimer 6 is produced by the elimination of the $R$ moiety by the attack of a nucleophile.

Cyclic dimer 6 consisting of a pair of the enantiomeric monomeric units has a center of symmetry and is crystallizable. Therefore, the precipitation of 6 which occurs immediately after it is formed must be one of the driving forces for its preferential formation irrespective of the energetically unfavorable, strained structure. When a monomer having the same chirality as that of the terminal unit of 9 attacks the acetal carbon of 9, the resulting bromonium ion 11 would not be able to cyclize to give a 10 -membered oxonium ion like 12 for a sterical reason. Presumably, such a bromonium ion either adds another monomer or depropagates.

In the oligomerization of $\mathbf{5 e}$, neighboring group participation of the equatorially located bromine atom is impossible, unless the oxonium ion is unimolecularly ring-opened to form an oxacarbenium ion. The electron withdrawing bromine atom disfavors such an unimolecular ring-opening reaction and therefore, the cyclic dimer cannot be formed by a similar mechanism to that described for the cyclodimerization of 5a. Probably a small amount of cyclic dimer of $5 \mathbf{e}$ as detected in the 
${ }^{1} \mathrm{H}$ NMR spectrum in Figure 3 is produced by a back-biting mechanism similar to that proposed previously for the formation of 2 in the oligomerization of 1.5 Such neighboring group participation of the axially oriented bromine atom was also postulated to explain the different behavior of the two stereoisomers of 4bromo-6,8-dioxabicyclo[3.2.1]octane in their cationic polymerization. ${ }^{19}$

In summary, the stereoisomers $\mathbf{5 a}$ and $\mathbf{5 e}$ displayed significantly different behavior in their cationic oligomerization at -40 and $0^{\circ} \mathrm{C}$. The axial isomer $\mathbf{5 a}$ had a tendency to cyclodimerize, particularly at higher temperature. The equatorial isomer 5e was much less reactive than 5a, and it afforded small amounts of cyclic oligomers. Their limitted solubilities, however, made it not feasible to determine the ring-size distribution by gel permeation chromatography. The specific formation of eyclic dimer 6 from 5a was reasonably interpreted in terms of the participation of bromonium ions in the propagation.

Acknowledgments. Financial support from the Ministry of Education, Science, and Culture of Japan (Grant-in-Aid for Scientific Research No. 59550622) is gratefully acknowledged. Thanks are also due to $\mathrm{Mr}$. S. Kitamura for assistance with the elemental analysis.

\section{REFERENCES}

1. J. A. Semlyen, Adv. Polym. Sci., 21, 43 (1976).
2. E. J. Goethals, Adv. Polym. Sci., 23, 103 (1977).

3. S. Penczek, P. Kubisa, and K. Matyjaszewski, $A d v$. Polym. Sci., 68/69, 1 (1985).

4. I. Tajima, M. Okada, and H. Sumitomo, Makromol. Chem., Rapid Commun., 1, 197 (1980).

5. I. Tajima, M. Okada, and H. Sumitomo, Macromolecules, 14, 1180 (1981).

6. M. Okada, H. Sumitomo, and M. Atsumi, Makromol. Chem., Rapid Commun., 4, 253 (1983).

7. M. Okada, H. Sumitomo, and M. Atsumi, J. Am. Chem. Soc., 106, 2101 (1984).

8. D. H. Haynes, T. Wiens, and B. C. Pressman, J. Membr. Biol., 18, 23 (1974).

9. I. Tajima, M. Okada, and H. Sumitomo, J. Am. Chem. Soc., 103, 4096 (1981).

10. M. Atsumi, M. Okada, and H. Sumitomo, Makromol. Chem., 188, 1137 (1987).

11. I. Tanaka, I. Tajima, Y. Hayakawa, M. Okada, M. Bitoh, T. Ashida, and H. Sumitomo, J. Am. Chem. Soc., 102, 7873 (1980).

12. M. Imaeda, I. Tanaka, T. Ashida, I. Tajima, M. Okada, and H. Sumitomo, Polym. J., 14, 197 (1982).

13. I. Sakuragi, I. Tanaka, T. Ashida, I. Tajima, M. Okada, and H. Sumitomo, J. Am. Chem. Soc., 104, 6035 (1982).

14. M. Okada, H. Sumitomo, M. Atsumi, H. K. Hall, Jr., and R. B. Ortega, Macromolecules, 19, 503 (1986).

15. M. Okada, H. Sumitomo, M. Atsumi, H. K. Hall, Jr., R. J. H. Chan, and R. B. Ortega, Macromolecules, 19, 953 (1986).

16. M. Okada, H. Sumitomo, M. Atsumi, and H. K. Hall, Jr., Macromolecules, 20, 1199 (1987).

17. W. H. Starness, Jr., F. C. Schilling, I. M. Plitz, R. E. Cais, and F. A. Bovey, Polym. Bull., 4, 552 (1981).

18. F. A. Jameison, F. C. Schilling, and F. A. Tonelli, Macromolecules, 19, 2168 (1986).

19. M. Okada, H. Sumitomo, and A. Sumi, Macomolecules, 15, 1238 (1982).

20. M. Okada, H. Sumitomo, and A. Sumi, Polym. J., 14, 59 (1982).

21. M. Okada, H. Sumitomo, and M. Atsumi, Macromolecules, 17, 1840 (1984). 